\title{
GÉNERO Y OPINIÓN. EL ROL SECUNDARIO DE LAS MUJERES EN LOS ESPACIOS PERIODÍSTICOS
}

\section{GENDER AND OPINION. SECONDARY ROLE OF WOMEN IN JOURNALISTIC TEXTS}

\author{
Miriam Suárez Romero. Universidad de Sevilla - España \\ miriamsuarez@us.es \\ Ana María Ortega Pérez. Universidad de Sevilla - España \\ Ana.ort.per@gmail.com
}

Resumen Este artículo realiza una revisión de las autorías de los textos opinativos, con especial énfasis en el papel que han tenido las mujeres en la producción de piezas de opinión en el diario español El País. Mediante la técnica metodológica del análisis de contenido se estudia si existe un determinante de género en las rutinas productivas de este periódico. Para ello, se analizan 2621 piezas publicadas en su edición digital durante cinco años (de 2012 a 2016), haciendo especial hincapié en la evolución de los aspectos formales y contextuales orientativos de la atribución de relevancia por parte del medio y la inclusión de las periodistas en este proceso de creación.

Palabras clave Opinión periodística, estereotipos de género, inclusión social, autoría, medios digitales.

\begin{abstract}
This article revises autorships of opinion texts, with special emphasis on the role that women have played in the production of opinion pieces in the most influential newspaper in Spain, El País. Through the methodological technique of content analysis, we study whether there is a gender determinant in the productive routines of this newspaper. To this end, 2621 pieces of their digital edition are analyzed during five years (2012 to 2016). Special emphasis is made on the evolution of formal and contextual aspects related to media relevance, as well as the inclusion of the journalists in this creation process.
\end{abstract}

Keywords Journalistic opinion, gender stereotypes, social inclusion, authorship, digital media.

\section{Relevancia del estudio de la estereotipación de género en el caso de la opinión}

Walter Lippmann (2003) articuló una extensa reflexión acerca del uso y función de los estereotipos, un mecanismo de simplificación y efectiva economización pues, si empleásemos siempre una mirada minuciosa, nos agotaríamos. No tenemos tiempo ni capacidad para conocer todo de forma detallada antes de realizar un juicio mental. Pastor Ruiz (2006, p.186) los define como "una generalización en las atribuciones 
sociales sobre una persona por causa de su pertenencia a un grupo determinado". Son mecanismos de defensa de nuestra posición en la sociedad que "constituyen una imagen ordenada y más o menos coherente del mundo, (...). Puede que no formen una imagen completa, pero son la imagen de un mundo posible al que nos hemos adaptado. En él, las personas y las cosas ocupan un lugar inequívoco y su comportamiento responde a lo que esperamos de ellos" (Lippmann, 2003, p.93).

La mentalidad a través de la que se filtra la información recibida, junto con la información de la que disponemos, conforma nuestra forma de pensar. Es un sistema cómodo y organizado, por lo que los intentos de desestabilización del orden de nuestro mundo son, como dice Lippmann, un ataque contra los mismísimos pilares del universo, porque son un ataque contra los pilares de nuestro universo.

Así lo explican también Sell e Imas (2014, p.86): durante el proceso de socialización establecemos categorías para ordenar el mundo, "atajos cognitivos que ayudan a las personas a tomar decisiones, basándose en lo que se conoce, y entiende como característico de su grupo". Al interactuar con una persona esperamos que se cumpla esa categorización según el grupo en el que la hemos incluido. Los estereotipos son, por tanto, el resultado de esta categorización social, creencias compartidas por un grupo. Su variante, el prejuicio, es la "evaluación preconcebida que se basa en una información limitada sobre un grupo o conjunto de personas" (2014, p.91). Los medios de comunicación, apuntan estos autores, han sido estudiados desde dos perspectivas definidas por Núñez Domínguez (2001, p.92) como el doble valor de los medios de comunicación:

El estudio de los medios de comunicación y su influencia como agentes sociales puede estructurarse desde dos enfoques en la psicología social: a) como reproductor de la realidad, asumiendo cómo sus narrativas y contenidos reproducen los estereotipos (Gerbner et al., 1996), y b) como generador de modelos, donde se asume que la audiencia es activa y está incorporando los contenidos y su estructura narrativa en su forma de ver la vida.

En la teoría del doble valor de los medios de comunicación radica la importancia del objeto de estudio de este artículo: los medios como reproductores de la realidad y generadores de modelos en la sociedad. La prensa orienta mediante el acercamiento a modelos preexistentes, con la consiguiente cercanía del lector que ello conlleva, pues se siente identificado con los estereotipos que se reproducen en el medio.

Cuando ya tenemos nuestro sistema de estereotipos consolidado, tendemos a concentrarnos en hechos que lo reafirman, pasando por alto los que no, y reforzándose lo que creemos congruente: "Los estímulos procedentes del exterior, especialmente cuando se transmiten de forma verbal o por medio de la letra impresa, evocan en cierta medida algunos aspectos de los sistemas de estereotipos. De esta manera, percepciones sensoriales e ideas preconcebidas se mezclan y alojan en nuestra conciencia de forma simultánea, (...)" (Lippmann, 2003, p.96). 
El caso que nos ocupa se refiere a unos estereotipos muy concretos (los de género) y en un lugar delimitado (la prensa de opinión). Son numerosas las publicaciones que han abordado las relaciones existentes entre los medios de comunicación y las mujeres a lo largo de la historia, con una producción muy fructífera en los años go que se centra principalmente en su papel protagonista, es decir, cómo son representadas (Landázuri, 1990; Valdivia, 1990; Pérez Infante y Martínez Ten, 1991; Valle Ferrer et al., 2003).

Inmersos en un contexto de periodismo altamente digital como forma de comunicación social, esta investigación se marca un objetivo diferente: observar la participación de la mujer como profesional del periodismo, concretamente como creadora de opinión. Los textos de opinión son uno de los pilares de cualquier medio de comunicación, reflejo de la ideología del grupo empresarial que lo sostiene. Todo periódico pertenece a una empresa a la que ha de rendir cuentas, más allá de la función social que se le presuponga.

En la lógica empresarial de atraer lectores, los artículos de los diarios se redactan para suscitar atención. El mantenimiento del interés será uno de los propósitos fundamentales de los textos de opinión. León Gross (1996, p.197) recalca la constante actividad propagandística del que opina, que usa mecanismos válidos desplegados para la consecución de un fin: "la persuasión de un auditorio a propósito de algún aspecto de cierta relevancia en ese magma heterogéneo que constituyen los asuntos de interés general que se producen o al menos se dan a conocer al albur de su valor de actualidad". Pero esta persuasión en los textos de opinión no es agresiva, pues satisface al lector. El artículo confirma lo que creía de antemano, y además ve su suposición respaldada por un líder de opinión, por lo que ahora su opinión tiene aún más fundamento y prestigio. Se cumplen así las funciones atribuidas tradicionalmente al periodismo: informar, deleitar, orientar.

El rol y la capacidad de influencia del líder de opinión de un medio de comunicación de alta relevancia no es baladí. Deriva de ello la necesidad de conocer la situación y el rol de las mujeres en este campo. Se estudian aquí las autoras de la sección de opinión del periódico El País durante seis años. Las mujeres han estado durante mucho tiempo relegadas a secciones como sociedad, belleza y salud. Temas como economía, política o medio ambiente, al igual que la mayoría de los altos mandos de los medios, estaban en manos de hombres (Glattsein Franco, 1995, p.75).

Centrándonos en el rol que nos ocupa, el de la producción, es evidente que las redacciones están ocupadas por un gran número de mujeres. La situación ha cambiado con el paso de los años, pero no se ha alcanzado una igualdad efectiva en absoluto. Uno de los pocos estudios referentes a la mujer como creadora de opinión lo encontramos en Gènere e Informació de 1998 (Bach Arús et al., 2000, p.66). De acuerdo a su estudio, únicamente el 7,04\% de los artículos de opinión están firmados por mujeres frente al $69,62 \%$ que firman hombres, desigualdad más amplia incluso 
que en información, en el que el $12,40 \%$ de autoría pertenece a mujeres y un $27,84 \%$ a hombres. Si bien ha habido cambios desde este estudio de 1998, no son tan radicales como deberían.

En abril de 2013, el estudio "Igualdad en el periodismo" ${ }^{1}$ promovido por la Federación de Asociaciones de Periodistas de España determinaba que un 45\% de la muestra total encuestada entendía que seguían dándose "prácticas discriminatorias de algún tipo hacia la mujer en la profesión periodística. Un $57 \%$ de las mujeres y un $28 \%$ de los hombres subrayan esa opinión" (2013, p.4). Los encuestados no mostraron demasiada preocupación en ello: "La importancia media que tiene favorecer el principio de igualdad, en aspectos como la objetividad editorial, la deontología, las relaciones internas o la relaciones con la audiencia, no alcanzó en ningún caso la calificación de cuatro (de o a 5)", y las mujeres (61\%) creen en mayor proporción que los hombres (42\%) que en las empresas no se respeta el principio de igualdad.

Las mujeres siguen estando lejos de ser cuantiosas como líderes de opinión en los diarios de referencia, y es un problema de desigualdad mucho más grave de lo que es percibido en los anteriores sondeos.

El Informe Mundial sobre la Condición de la Mujer en los Medios de Comunicación de la UNESCO (2011) $)^{2}$ mostraba que:

$>$ Los hombres ocupan la gran mayoría de los escaños en los consejos rectores y en la alta dirección en siete regiones, en un $74,1 \%$ y $72,7 \%$, respectivamente.

> Los hombres están en casi el 71,3\% de los puestos de jerarquía intermedia de las empresas encuestadas, y las mujeres en el $28,7 \%$ restante.

> El 61,3\% de los cargos de alta dirección son ocupados por hombres (un 38,7 \% en el caso de mujeres).

> El nivel subalterno del cuadro orgánico está dominado por los hombres, con una proporción de casi el 63,9\%, mientras que las mujeres desempeñan el 36,1 $\%$ de dichos cargos.

$>$ En el área profesional técnica, los hombres poseen el 73,2\% de los empleos y las mujeres apenas un $26,8 \%$.

$>$ Solo un $16 \%$ de las empresas mediáticas observadas en Europa Oriental, un 27 $\%$ en la región de Oriente Medio y África del Norte y un $69 \%$ en África subsahariana posee una política de empresa sobre la igualdad de género.

\footnotetext{
${ }^{1}$ Puede consultarse en: <http://www.estudiodecomunicacion.com/extranet/wpcontent/uploads/2013/04/ESTUDIO\%20DE\%20COMUNICACI\%C3\%93NMFAPE\%2olgualdad\%2operiodismo.pdf> ${ }^{2}$ Disponible en: <http://www.unesco.org/new/es/communication-and-information/crosscutting-
priorities/gender-and-media/women-make-the-news/facts-and-figures/>
} 
En el diario Público, Torrús (2016) publicaba el artículo "El techo de acero de las mujeres" ${ }^{\prime \prime}$, en el que exponía, basándose en el informe de la Asociación de la Prensa de Madrid, que las profesionales sólo ocupan el 10,9\% de los puestos de dirección en los medios impresos, el 7,8\% en los audiovisuales y sólo el 3,9\% de los responsables de contenidos en los medios digitales son mujeres".

Ante estas cifras, consideramos relevantes los estudios que ahonden en el papel de la mujer en determinados ámbitos en los que ha estado relegada tradicionalmente a un segundo plano. Es el caso, como se trata en este estudio, de los prestigiosos espacios de opinión de los medios de comunicación.

\section{Diseño metodológico}

A la luz de los datos aportados anteriormente, el objetivo de este artículo es estudiar la presencia de las mujeres como creadoras de opinión en el diario El País. Se establecen las siguientes hipótesis:

H1. Son minoritarias las piezas opinativas escritas por mujeres en la edición digital de El País.

H2. Las opiniones firmadas por mujeres no presentan en general aspectos formales que indiquen una atribución de importancia por parte del periódico.

H3. Ha habido una evolución cuantitativa y cualitativa en el rol de la mujer en la rutina de producción de opinión desde 2012 a 2016 en el diario estudiado.

Para testar estas hipótesis se emplea una metodología cuantitativa. No obstante, los valores, normas y definiciones situacionales de nuestra sociedad son tenidos en cuenta a la hora de sistematizar las propiedades del texto al que se aplica la técnica metodológica de esta investigación, el análisis de contenido. Su práctica no permite predecir fenómenos ni generar reglas universales (Raymond Colle, 2011), pero el propósito del uso en este artículo es formular inferencias y estudiar las frecuencias de los ítems recogidos en la ficha y el libro de códigos ideados específicamente para el estudio propuesto.

La ficha de análisis diseñada a tal efecto contiene el nombre del codificador, titular de la pieza, fecha de aparición/publicación, número de codificación, año de publicación (2012-2016), género periodístico de opinión (artículo, columna, editorial, tribuna, critica o análisis), temática principal (política internacional, política nacional, política regional/local, sociedad/sucesos, economía, deportes, comunicación/tecnología, cultura u otros temas), extensión en palabras (de o a 500, de 501 a 1000, de 1001 a 1500 o más de 1500) y autoría (no aparece el autor, hombre, mujer, hombre y mujer, agencias o El País).

\footnotetext{
${ }^{3}$ Disponible en: <http://www.publico.es/sociedad/gigantesco-techo-cristal-mujeres-periodismo.html>
} 
Si bien el objeto principal de estudio son las autorías, Mayntz, Holm y Hübner (1993, p.198) nos recuerdan que la naturaleza sistemática de la técnica que estamos aplicando exige que se tengan en cuenta todos los aspectos relevantes del texto para un problema de investigación, puesto que, de lo contrario, puede caerse en el error de registrar características confirmadoras de las hipótesis previamente planteadas. De ahí la elección de los aspectos formales de la ficha de análisis.

Los cuatro primeros ítems de la ficha son de reconocimiento. Los restantes, y mediante el uso de un libro de códigos, recogen aspectos formales para estudiar la evolución temporal, qué tipos de piezas firman las mujeres, en qué sección escriben o cuánto espacio ocupan, en relación al corpus.

Por su parte, el corpus está constituido por los textos de opinión de la edición digital de El País, uno de los grandes diarios del panorama mediático español, desde 2012 a 2016, ambos inclusive. Se ha seleccionado este periódico por ser el diario online de información general con más visitantes únicos de los sitios de internet controlados por el Estudio General de Medios (AIMC) para todas las oleadas y años móviles tenidos en consideración. La selección de los días se ha realizado mediante muestreo aleatorio, tomando los periódicos publicados cada 15 días en todos los años. Han sido tenidos en cuenta para el análisis los siguientes géneros: artículo de opinión, columna, editorial, tribuna, crítica y análisis ${ }^{45}$.

\section{Resultados y discusión}

La muestra resultante de la selección efectuada ya comentada está compuesta por 169 días de análisis completos. En total, el corpus está formado por 2621 piezas de opinión, repartidas como sigue:

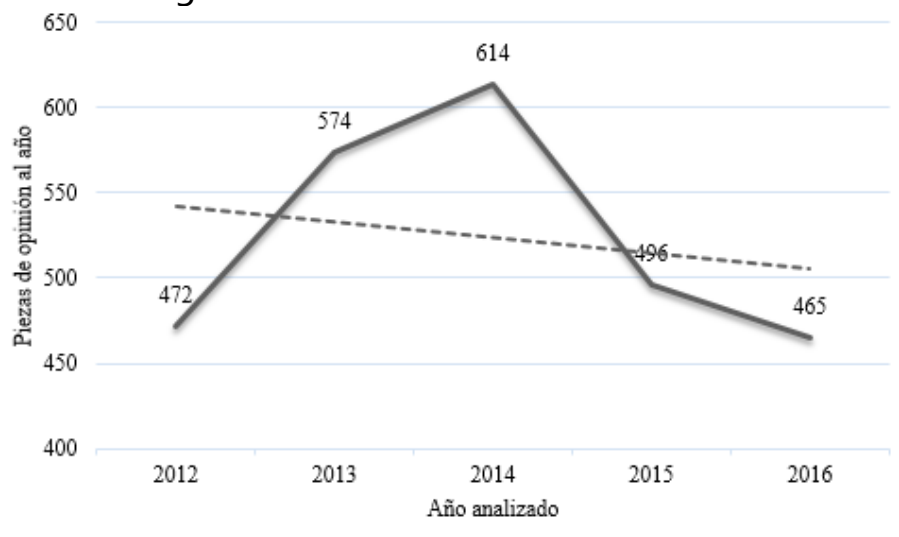

\footnotetext{
${ }^{4}$ No han sido contemplados blogs personales de los periodistas que están hipervinculados, ni viñetas, ante las diferencias formales con el resto de piezas y la consecuente incapacidad de aplicación de la ficha de análisis tipo diseñada.

${ }^{5}$ En El País hemos encontrado contadas excepciones en las que los contenidos no son renovados completamente cada día. Esto hace que el periódico que se le presenta al usuario un día concreto tenga textos que pertenecen a días anteriores. Para estudiar de una forma fidedigna lo publicado, se han tenido en cuenta también estas piezas fechadas en días anteriores, puesto que son ofrecidas al lector como parte de la información del día, y conforman la actualidad que el ciudadano consulta y que el periódico le brinda.
} 
Figura 1. Evolución cuantitativa de las piezas de opinión publicadas desde 2012 a 2016. Fuente: Elaboración propia

Comentamos primeramente los resultados totales, para posteriormente centrarnos en los datos relativos a la mujer como creadora de opinión en este estudio.

2014 es el año más cuantioso en lo que a producción se refiere, y 2016 el menos. La línea de tendencia, como puede observarse en el gráfico con un resaltado de puntos, presenta una evolución descendente. Los resultados totales tienen una forma evidente de valle, con el pico máximo en las 614 piezas de opinión con las que cuenta 2014, que aglomera el $23,42 \%$ de todo el corpus.

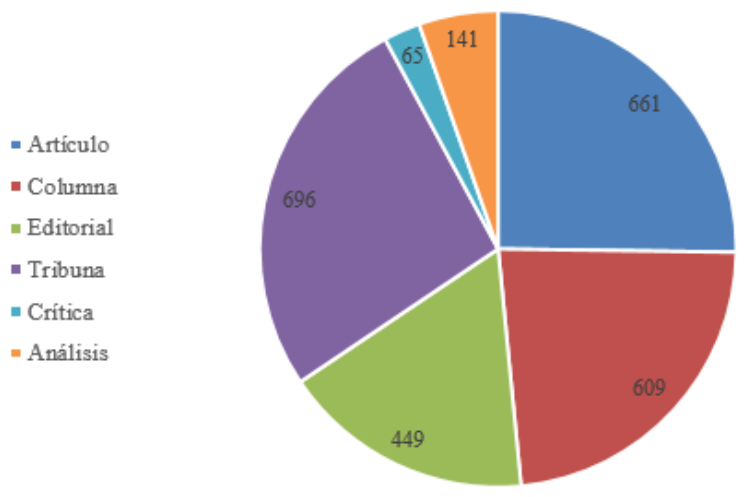

Figura 2. Géneros de opinión. Fuente: Elaboración propia.

En lo referente a los géneros, predominan las tribunas $(26,55 \%)$, un género donde las firmas de los autores son tenidas en especial consideración por su relevancia en el campo al que pertenecen. Están seguidas por los artículos $(25,22 \%)$. Las menos numerosas son las críticas $(2,48 \%)$, que constituyen un género mixto o híbrido por su carácter informativo y su función de orientación de la opinión.

El siguiente ítem de la ficha de análisis nos permite observar en qué sección están incluidas las producciones de opinión en El País.

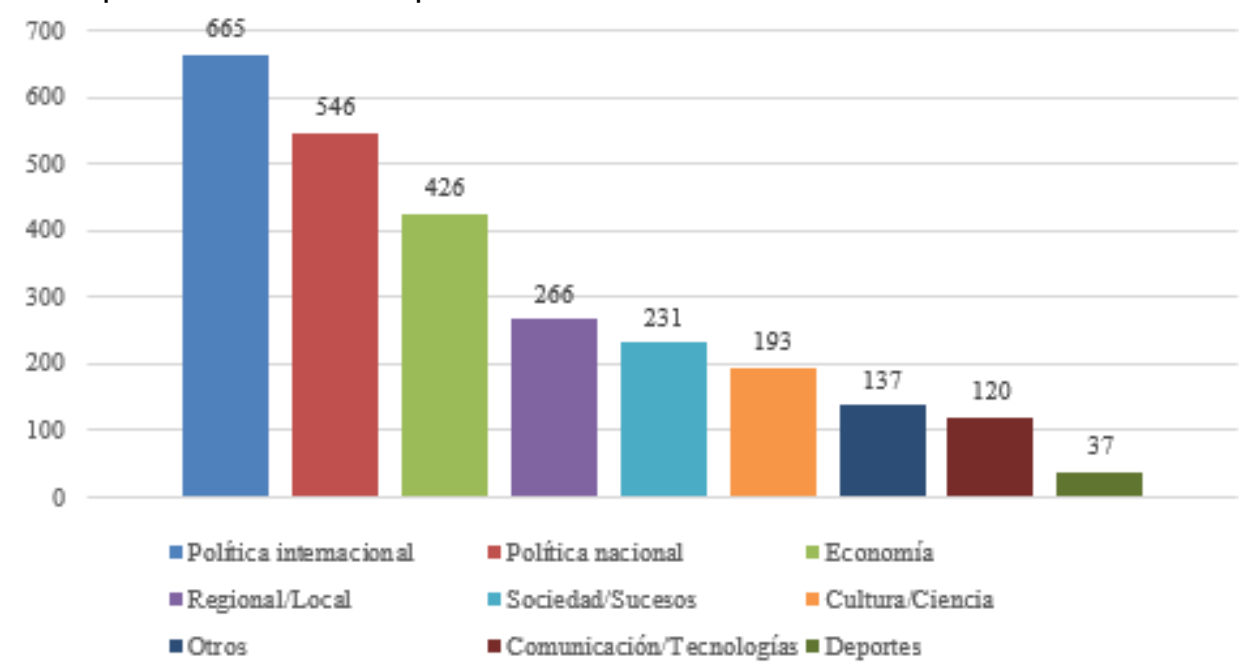

Figura 3. Secciones del periódico en las que está incluidas las unidades analizadas. Fuente: Elaboración propia 
Destacan claramente las secciones de política internacional (25,37\%), seguida de política nacional $(20,83 \%)$. Ambos constituyen espacios con gran cabida para estos tipos de textos y, como se esperaba, contienen mucha más opinión que el resto de secciones, ya que ellas dos juntas albergan casi la mitad de lo publicado. Entre las secciones con menos opiniones publicadas están deportes $(1,41 \%)$ y comunicación $(4,58 \%)$.

Otro de los parámetros medidos era la extensión, en caracteres, de las unidades seleccionadas. La medida más común es entre 501 y 1000 caracteres: 1152 (un 43.95\% del total de las 2621 piezas constituyentes del corpus). Le siguen los artículos más breves, es decir, aquellos que cuentan con entre o y 500 caracteres, con 841 (32\%), los de 1001 a 1500 (602, un 23\%) y, por último, los más extensos, los de más de 1500, que tan solo se han encontrado en 26 ocasiones.

La variable más importante, sin embargo, para los propósitos de este estudio, es aquella relativa al género de la persona que las escribe. Pasamos así a ahondar en los artículos firmados por mujeres, para tratar de esbozar un perfil más detallado de la función que han tenido en estos espacios tan determinantes del diario durante el último lustro.

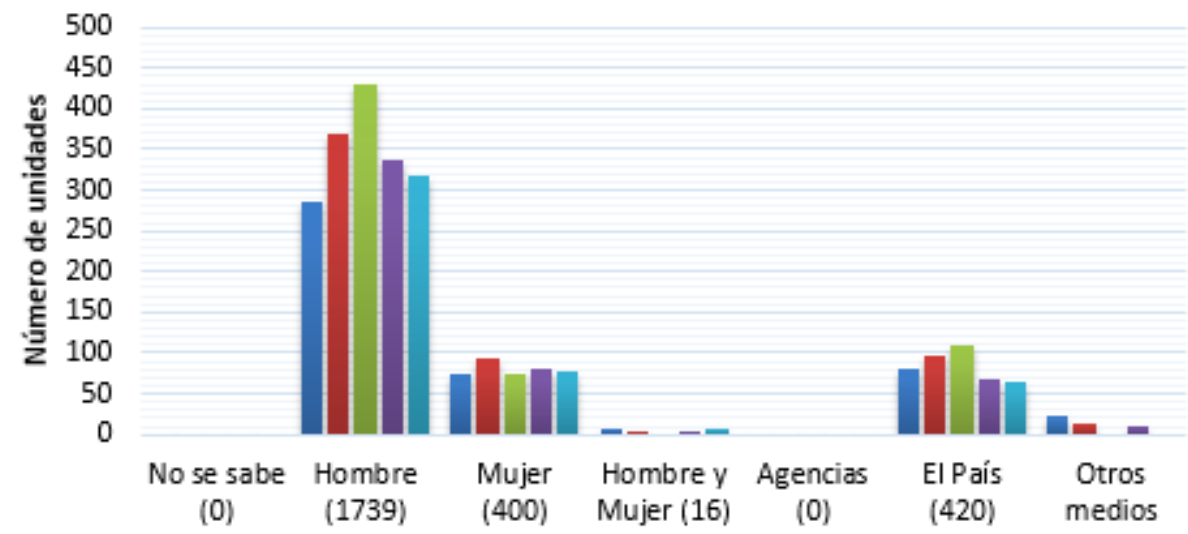

(46)

Autores (número total de piezas en los cinco años de estudio)

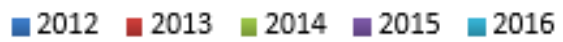

Figura 4. Reparto de la autoría del conjunto de las piezas de opinión analizadas.

Fuente: Elaboración propia.

Claramente, las mujeres están relegadas a un plano posterior en la creación de opinión de El País. Resulta sorprendente que un 66,35\% de todo el corpus analizado, 2621 unidades durante cinco años, tenga como autor a un hombre. Una cifra altamente significativa si tenemos en cuenta que el porcentaje restante hasta el total no es, ni mucho menos, de producción femenina. De hecho, hasta el propio periódico supera a las mujeres cuantitativamente hablando, con 420 piezas que suponen un $16 \%$ del total. No hay ninguna pieza cuya autoría no esté especificada ni ninguna manifiestamente de agencias. 16 piezas tienen autoría compartida entre ambos géneros. 
Cabe recordar que se han analizado 169 días completos del periódico digital durante cinco años, seleccionados mediante muestreo aleatorio sistemático. Las mujeres han firmado en todo este tiempo representado un total de 400 piezas, de las 2621 que constituyen la muestra, un $15,26 \%$. Las mujeres no son, en definitiva, creadoras de opinión en este periódico de referencia nacional. Esta es la conclusión que arroja un primer análisis cuantitativo del estudio.

Si nos detenemos a observar los datos por años, tal y como se presentan en el gráfico anterior, vemos cómo no hay ningún año que presente cifras por encima del resto de años, y están todos más o menos igualados. Sí que destaca claramente en $E l$ País las barras correspondientes a la autoría masculina, y en concreto las correspondientes a 2013 y 2014. Debe ser recordado que son los años con más producción numérica de piezas de opinión (574 en 2013 y 614 en 2014; 2012, 2015 y 2016 no alcanzan ninguno las 500 unidades), lo que parece haber supuesto un incremento en la producción de opinión de autores e incluso de piezas firmadas por el propio periódico, pero no se ha traducido en un aumento de espacios para firmas de mujeres. Las cifras son, en definitiva, igual de mediocres en todos los años. Si aislamos las firmas de mujeres y hombres, el resultado habla por sí mismo:

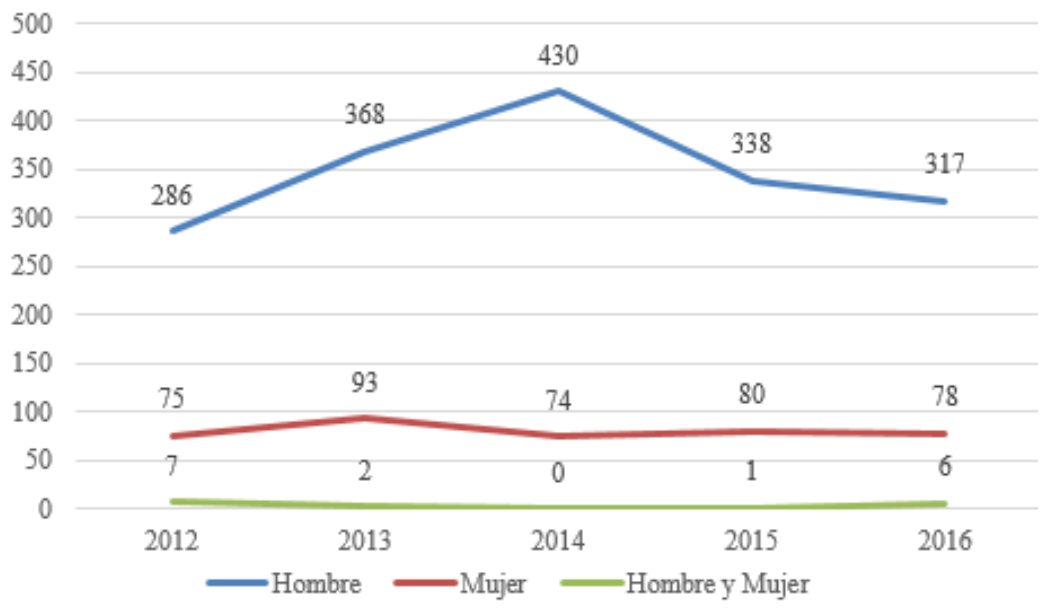

Figura 5. Número de espacios de opinión en El País firmado por mujeres y hombres. Fuente: Elaboración propia.

No solo es cuantiosamente superior, sino que incluso tiene picos de producción, algo que no pasa en el caso de las mujeres, que se mantienen en una línea casi plana de escasez. Para profundizar en el perfil de lo publicado por las mujeres en este diario, -asociándolo a criterios de atribución de importancia - , la variable relativa al género es mostrada en la siguiente gráfica, en la que se han aislado las 400 unidades que presentan una firma femenina, y se ha dividido por años y géneros. Las columnas son, con diferencia, las más utilizadas, con 183 piezas que suponen el $45,75 \%$ de todo lo publicado por ellas, un género personalista con características como la asiduidad o la regularidad de la publicación, una ubicación fija y la extensión más corta de todos los géneros tipificados en esta variable. Le sigue el artículo, con un $28,5 \%$ de todo lo escrito y, en mucha menor medida, la tribuna (68), el análisis (19) y la crítica (14). 


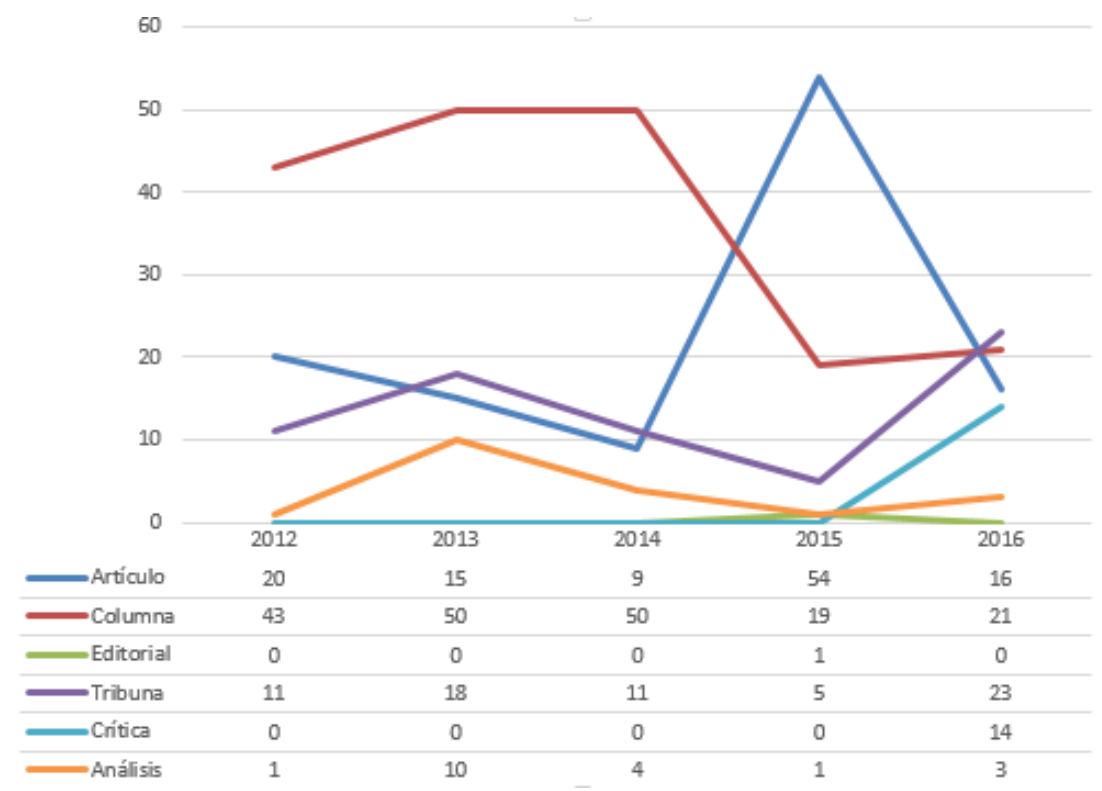

Figura 6. Producción por géneros periodísticos de las mujeres en El País. Fuente: Elaboración propia.

Varían también las producciones según los años. Como puede observarse en el gráfico superior conforme ha avanzado el tiempo se han publicado más artículos firmados por mujeres y han caído notablemente las columnas. El resto de géneros presenta altibajos pero siempre dentro de los mismos márgenes, sin casi sobrepasar las 20 unidades en ninguno de los años.

La baja atribución de importancia reflejada por la poca extensión del género más asociado a las publicaciones de las periodistas tiene una continuidad en los caracteres de lo que han escrito, si bien al menos no predominan claramente las piezas más pequeñas tipificadas. Lo hace el segundo nivel, de 501 a 1000 caracteres, con un $47,25 \%$ de todas las piezas analizadas firmadas por mujeres. También era así en los datos generales sin diferenciar la autoría en los cinco años, representando un $43,95 \%$ del corpus total. Le siguen aquellas de o a $500(36,75 \%)$ y, con bastante distancia, las de 1001 a 1500 (15,25\%), siendo las de más de 1500 caracteres casi inexistentes y representadas por tres piezas. Además de los datos conjuntos, en la siguiente figura puede apreciarse la evolución numérica por años: 


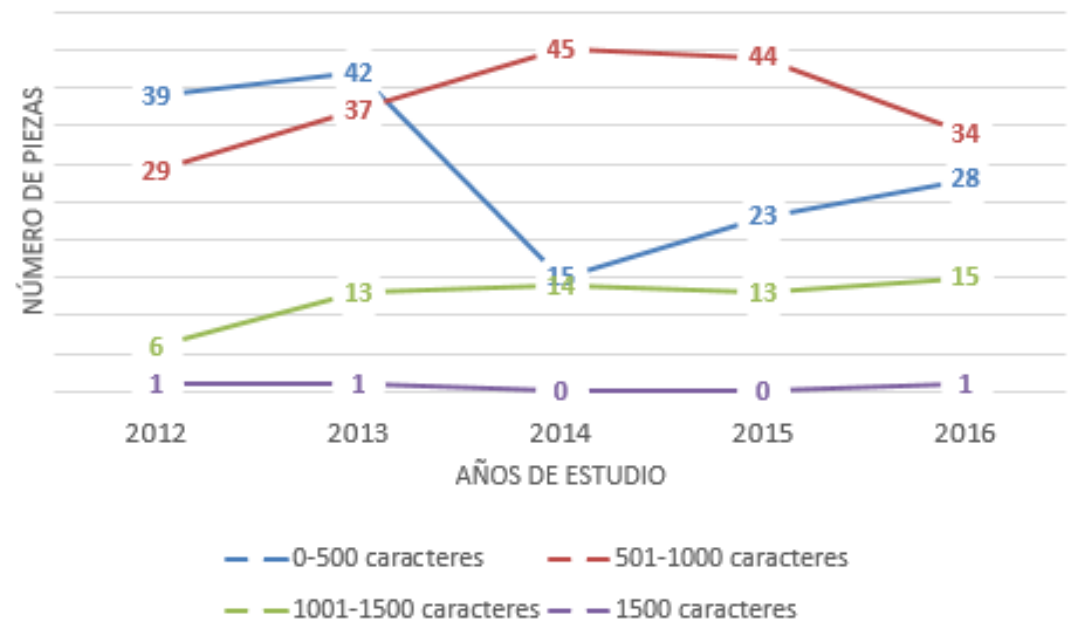

Figura 7. Número de caracteres de las opiniones de las mujeres autoras en El País, divididas por años. Fuente: Elaboración propia.

Las dos primeras cuantificaciones de la escala, las menores, además de ser las más cuantiosas, son las que más fluctúan en todo el periodo, sobre todo el primer tramo (0-500), que presenta una bajada considerable en 2014, compensada por el segundo tramo (501-1000), como puede deducirse de la observación del gráfico arriba presentado. La última de las variables destacadas para conocer el perfil de publicación buscado son las secciones en las que se incluye toda esta producción.

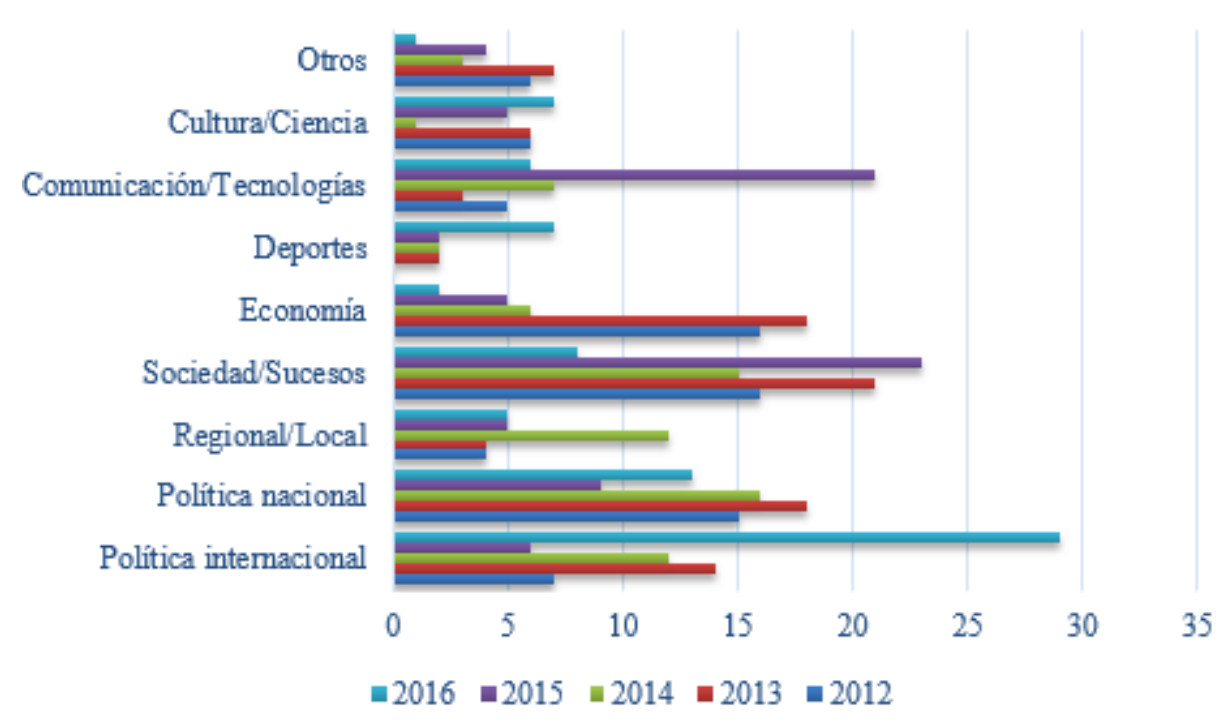

Figura 8. Comparativa de la distribución del número de piezas publicadas en las secciones en las que escriben las autoras en los cinco años.

La sección más recurrida es Sociedad/Sucesos, con un $20,75 \%$ de todo lo publicado. Es el apartado más generalista y amplio de todos los tipificados, e incluye aspectos tan variopintos como educación, terrorismo, medio ambiente, sanidad o problemas sociales, entre otros. En el análisis de los datos conjuntos sobre la muestra total de 2621 unidades, las secciones sobresalientes eran política internacional $(25,37 \%)$ y nacional $(20,83 \%)$. Son también, aunque en orden inverso, las siguientes 
con los resultados filtrados por la autoría femenina, pues a Sociedad le sigue en orden decreciente la política nacional (17,75\%) e internacional (17\%). En el otro extremo de la lista encontramos las menos cuantiosas, que son Deportes $(3,25 \%)$, Otros $(5,25 \%)$ y Cultura/Ciencia $(6,25 \%)$.

Estos son, sin embargo, datos conjuntos tomando toda la muestra. Si nos fijamos en los años por separado, las proporciones varían. Los cambios más acusados están presentes en la sección de política internacional, en la que hay un incremento considerable en 2016, último año de estudio, y en economía, donde los dos primeros años (2012 y 2013) triplican en número a los restantes registrándose una considerable disminución de las piezas firmadas por las mujeres en este periodo. También ocurre en la sección relativa a Comunicación/Tecnologías, con un pico sobresaliente en 2015 que se repite en la sección Regional/Local en 2014.

\section{Conclusiones}

Este estudio pretendía hacer una breve aproximación al rol del género en los procesos de producción periodística de opinión, a través del análisis de sus autorías. Se partía para ello de una primera hipótesis de que las piezas opinativas escritas por mujeres en la edición digital de El País eran minoritarias. Efectivamente, tras analizar 169 días completos del periódico digital, las mujeres han firmado de 2012 a 2016 un total de 400 piezas de las 2621 que constituyen la muestra, lo que representa un $15,26 \%$ del conjunto. Son superadas incluso por las piezas de opinión que el propio periódico publica y, por supuesto, por sus compañeros, que con 1739 unidades, firman el $66,35 \%$ del corpus. También presuponíamos que las opiniones publicadas por mujeres no presentaban en general aspectos formales que indicasen una atribución de importancia por parte del periódico, algo que ha sido evidenciado a través de la constatación del uso mayoritario de espacios pequeños como son las columnas, y medidas en caracteres poco cuantiosas.

Sin embargo, los datos presentados constatan que no ha habido una evolución cuantitativa ni cualitativa en el rol de la mujer en la rutina de producción de opinión desde 2012 a 2016 en el diario estudiado. Las mayores diferencias por años se han registrado en las líneas temáticas. Pero, si bien el resto de variables tenidas en cuenta en este estudio para el análisis de contenido solo varían por criterios estrictamente profesionales del medio (que El País le de un espacio mayor o menor a la autora o que le inste a publicar la opinión siguiendo un género periodístico marcado previamente, por ejemplo), no ocurre así en la sección de inclusión. En este caso, las proporciones presentadas dependen en gran parte de la actualidad del momento. Sirva como ejemplo 2015, que cuenta con unas elecciones generales en España y un proceso independentista catalán como temas de máxima actualidad, incrementándose así las piezas nacionales y regionales, e incluso otras dedicadas a principio de año a temas sobre terrorismo por el atentado contra Charlie Hebdo.

Aunque estos aspectos puedan variar levemente las secciones predominantes, se ha observado una tendencia común en todos los años de estudio: las mujeres, además de firmar pocas piezas, son siempre las mismas. No hay un gran número de autoras de opinión en este periódico, quedando relegadas a un segundo plano. Por ejemplo, un 
monto importante de esas 400 firmas femeninas son "Defensoras del Lector ${ }^{6}$ ", y no hay representación de mujeres en los grandes espacios que el periódico reserva a invitados ilustres, como son las tribunas, que además son los textos más extensos. Desafortunadamente, también en la plantilla del periódico se mantiene esta tendencia. Basta con acceder al apartado "Nuestras firmas" de su página web7. De 51 nombres que contiene el listado, tan solo 10 son mujeres: Soledad Gallego Díaz, Leila Guerriero, Almudena Grandes, Sandra León, Elvira Lindo, Valeria Luiselli, Máriam Martínez-Bascuñán, Rosa Montero, Yoani Sánchez y Luz Sánchez-Mellado.

En líneas futuras de estudio, podría ampliarse esta indagación a otros periódicos nacionales e internacionales para comprobar si se trata de una tendencia general en la producción periodística de opinión $y$, en tal caso, ser conscientes de que es una desigualdad social relevante de la que debiéramos ser conscientes para poder promocionar un cambio paulatino en las rutinas de los medios actuales, motor creador y reproductor de las tendencias y corrientes de opinión dominantes en nuestra sociedad.

\section{Referencias bibliográficas}

Bach Arús, M. et al. (2000). El sexo de la noticia. Reflexiones sobre el género en la información y recomendaciones de estilo. Barcelona: Icaria Editorial

Colle, R. (2011). "El análisis de contenido de las comunicaciones". 1-Fundamentos. Cuadernos artesanos de Latina, 11. La Laguna, Tenerife: Sociedad Latina de Comunicación Social. Disponible en: <http://www.revistalatinacs.org/o67/cuadernos/13_Colle_interior.pdf>

Federación de Asociaciones de Periodistas de España (2013). Estudio "Igualdad en el periodismo". Abril de 2013. Disponible en: < https://goo.gl/kcEmt3 >

Ferrer, N.V.; Amado, A.M. y Hiriart, B. (2003). "Propuestas concretas para aproximarnos a un periodismo no sexista" en Medios de comunicación y violencia contra las mujeres, 2003, pp.255-270.

Glattstein Franco, S. (1995). "La mujer como objeto y sujeto de la comunicación" en Las mujeres en la opinión pública: X Jornadas de investigación interdisciplinaria sobre la mujer, Instituto Universitario de Estudios de la Mujer, Universidad Autónoma de Madrid.

Landázuri, M. (1990). "La mujer en la prensa". Chasqui: Revista Latinoamericana de Comunicación, 34, pp. 33-36.

León Gross, T. (1996). El artículo de opinión: introducción a la historia y la teoría del articulismo español. Barcelona: Ariel.

Lippmann, W. (2003). La opinión pública. Madrid: Cuadernos de Langre.

Mayntz, R., Holm, K., y Hübner, P. (1993). Introducción a los métodos de la sociología empírica. Madrid: Alianza.

Núñez Domínguez, T. (2014). Competencias psicosociales para profesionales de los medios. Madrid: Pirámide.

\footnotetext{
${ }^{6}$ Así define El País este puesto: La figura del Defensor del Lector fue creada por la Dirección de EL PAIS para garantizar los derechos de los lectores, atender a sus dudas, quejas y sugerencias sobre los contenidos del periódico, así como para vigilar que el tratamiento de las informaciones es acorde con las reglas éticas y profesionales del periodismo. Puede intervenir a instancia de cualquier lector o por iniciativa propia. Puede consultarse en: < http://blogs.elpais.com/defensor-del-lector/2017/02/21/>

${ }^{7}$ Puede consultarse en la siguiente dirección: <http://elpais.com/elpais/firmas.html>
} 
Pastor Ruiz, Y. (coord.). (2006). Psicología social de la comunicación: aspectos básicos y aplicados. Madrid: Pirámide.

Pérez Indante, J. I. y Martínez Ten, C. (1991). Políticas para la igualdad de oportunidades de la mujer en España. Economía y sociología del trabajo, 13, pp.156-164.

Sell Trujillo, L., e Imas, M. (2014). "Eliminar los prejuicios en los medios" en Competencias psicosociales para profesionales de los medios (pp. 85-94). Madrid: Pirámide.

Torrús, A (2016). El techo de acero de las mujeres. Diario Público, 28 de febrero de 2016. Disponible en: < http://www.publico.es/sociedad/gigantesco-techocristal-mujeres-periodismo.html >

UNESCO (2011). Informe Mundial sobre la Condición de la Mujer en los Medios de Comunicación de la UNESCO. Disponible en: < http://www.unesco.org/new/es/communication-and-information/crosscuttingpriorities/gender-and-media/women-make-the-news/facts-and-figures/>

Valdivia, A.N. (1990). "Prensa, mujer y revolución". Chasqui: Revista Latinoamericana de Comunicación, 34, pp.45-47.

Cómo referenciar este artículo/How to reference this article:

Suárez Romero, M.; Ortega Pérez, A. (2019). Género y opinión. El rol secundario de las mujeres en los espacios periodísticos. iQUAL. Revista de Género e Igualdad, 2, 133-146, doi: 10.6018/iQual. 324321

Suárez Romero, M.; Ortega Pérez, A. (2019). Género y opinión. El rol secundario de las mujeres en los espacios periodísticos. [Gender and opinion. Secondary role of women in journalistic text]. iQUAL. Revista de Género e lgualdad, 2, 133-146, doi: 10.6018/iQual.324321 